REVISTA BRASILEIRA DE ANÁLISE DO COMPORTAMENTO / BRAZILIAN JOURNAL OF BEHAVIOR ANALYSIS, 2014, Vol. 10, Nº. 2, $175-186$.

\title{
DISCRIMINAÇÃO SIMPLES ENTRE ESTÍMULOS VISUAIS E REVERSÕES SUCESSIVAS DE DISCRIMINAÇÃO EM ABELHAS (MELIPONA QUADRIFASCIATA)
}

\author{
SIMPLE DISCRIMINATION AND SUCCESSIVE REVERSALS WITH VISUAL STIMULI IN BEES (MELIPONA \\ QUADRIFASCIATA)
}

ANTONIO MAURÍCIO MORENO

FACULDADE DE ILHÉUS, BRASIL

JULIA ZANETTI ROCCA

UNIVERSIDADE FEDERAL DO MATO GROSSO dO SUL, BRASIL

DEISY DAS GRAÇAS DE SOUZA

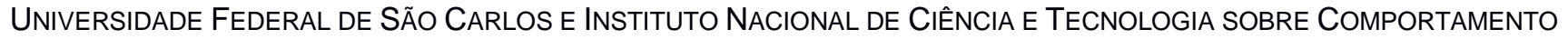
COGNIÇÃO E ENSINO, BRASIL

\section{RESUMO}

Com exceção dos estudos pioneiros de Isaías Pessotti sobre aprendizagem discriminativa em abelhas, outros trabalhos frequentemente empregaram procedimentos com características que dificultam o controle experimental rigoroso e uma interpretação segura dos dados (por exemplo, definição ambígua da resposta, treino simultâneo de vários sujeitos e análise de dados agrupados). Além disso, poucos estudos investigaram a aprendizagem discriminativa em outras espécies além de Apis mellifera e Bombus terrestris. O objetivo do Experimento 1 foi estabelecer discriminações simples entre cores e entre padrões em preto e branco em vinte abelhas individuais, da espécie Melipona quadrifasciata. No Experimento 2, o objetivo foi estabelecer discriminações simples e reversões de discriminação com quatro melíponas individuais em equipamento automatizado e pressão à barra como resposta operante. Todas as abelhas aprenderam a discriminar entre cores no Experimento 1, mas os dados de discriminação entre padrões em preto e branco mostraram grande variabilidade. No Experimento 2, as quatro abelhas aprenderam a discriminação inicial e também aprenderam entre uma e 11 reversões de discriminação. Os resultados de ambos os experimentos confirmam que melíponas podem aprender tarefas discriminativas, incluindo reversões sucessivas, mas também sugerem que os desempenhos podem variar consideravelmente a depender dos estímulos a serem discriminados.

Palavras-chave: discriminação simples, reversão de discriminação, estímulos visuais, abelhas, Melipona quadrifasciata

\section{ABSTRACT}

Except for the pioneering work of Isaías Pessotti on discriminative learning in bees, other studies often employed procedures that hinder rigorous experimental control and make difficult the data interpretation (e.g., ambiguous definition of the response, simultaneous training of various subjects, and analysis of pooled data). Moreover, few studies have investigated the discriminative learning in species other than Apis mellifera and Bombus terrestris. The purpose of Experiment 1 was to establish simple discrimination between colors and between black and white patterns, in twenty individual bees of the species Melipona quadrifasciata. In Experiment 2, the objective was to establish simple discrimination and discrimination reversals with four individual bees using electro-mechanical equipment and a bar press response. All the bees learned to discriminate between colors in Experiment 1, but discriminations between black and white patterns showed high individual differences. In Experiment 2, the four bees learned the initial discrimination and also learned from one to 11 discrimination reversals. The results of both experiments confirm that Meliponidae can learn visual discriminations, including successive reversals, but the data also suggest that performance may vary considerably depending on the discriminative stimuli.

Keywords: simple discrimination, reversal of discrimination, visual stimuli, bees, Melipona quadrifasciata

\footnotetext{
A pesquisa contou com financiamento da FAPESP como bolsa de mestrado para o primeiro autor. A redação do artigo foi apoiada pela FAPESP (Processo \#2008/57705-8) e pelo CNPq (Processo \# 573972/2008-7) com recursos concedidos ao Instituto Nacional de Ciência e Tecnologia sobre Comportamento, Cognição e Ensino (INCT-ECCE). Os autores agradecem ao professor Isaías Pessotti pelo legado sobre aprendizagem em abelhas. O trabalho é dedicado ao professor Luiz Marcelino de Oliveira pela incansável colaboração na adaptação e construção do equipamento automático. Enviar correspondência a Antonio Mauricio Moreno. Email: mauricio_amm@hotmail.com.
} 
Uma abordagem experimental da aprendizagem discriminativa em abelhas teve início ainda nas primeiras décadas do século passado, quando Karl von Frisch (von Frisch, 1914, 1967) desenvolveu um procedimento para estabelecer discriminação entre cores em abelhas da espécie Apis mellifera. Em um primeiro estudo (von Frisch, 1914), abelhas individualmente marcadas eram ensinadas a encontrar uma solução açucarada em um recipiente sinalizado com um cartão azul. Em uma fase de teste, o recipiente com cartão azul era posicionado entre recipientes marcados com cartões cinza, em diferentes tonalidades, mas semelhantes quanto ao brilho (podendo coincidir com o brilho do cartão azul). Uma vez que as abelhas consistentemente continuaram visitando o cartão azul, e não os cartões cinza, esse resultado foi uma demonstração de que as respostas de escolha ocorriam com base em percepção de cores, e não com base em algum mecanismo acromático.

Alguns aspectos experimentais do estudo pioneiro de von Frisch (1914) estão ainda presentes em muitos estudos sobre aprendizagem discriminativa em abelhas. Tipicamente, esses estudos são conduzidos em laboratórios nos quais uma ou mais colmeias são posicionadas junto a janelas da instalação, e algum aparato experimental é montado no interior do laboratório (e.g., Moreno, de Souza, \& Reinhard, 2012). Desse modo, as abelhas não ficam confinadas. As operárias podem voar para o ambiente externo, mas também podem aprender a voar até o interior do laboratório e receber uma solução açucarada, como estímulo reforçador de uma resposta de escolha, no contexto de uma tarefa discriminativa. No início de um experimento, um recipiente com solução açucarada é posicionado próximo à colmeia. Quando algumas abelhas são observadas recolhendo a solução, esse recipiente é gradualmente deslocado para o interior do laboratório (modelagem por aproximações sucessivas), até que algumas abelhas aprendam a encontrar a solução junto ao aparato experimental. Nesse momento, uma ou mais abelhas são marcadas para serem treinadas individualmente (e.g., Pessotti, 1981) ou em grupo (e.g., Giurfa, Zhang, Jenett, Menzel, \& Srinivasan, 2001). Após uma abelha sugar a solução açucarada até sua capacidade, ela retorna ao interior da colmeia, onde deposita a solução coletada. Em seguida, tipicamente, voa de volta ao aparato experimental onde aprendeu a coletar. Se a colmeia estiver posicionada a poucos metros da situação experimental, essa rotina de voar até o aparato, sugar a solução e voar de volta para colmeia pode ser completada em pouco mais de dois minutos, e se repetir regularmente, enquanto houver luz solar, desde o início da manhã até o final da tarde.

Uma extensa literatura mostrou que abelhas de várias espécies aprendem rapidamente a discriminar cores (e.g., Backhaus, 1993; Dyer, \& Chittka, 2004; Giurfa, Núñez, Chittka, \& Menzel, 1995; Giurfa, Vorobyev, Kevan, \& Menzel, 1996; Gumbert, 2000; Hempel de Ibarra, Giurfa, \& Vorobyev, 2002; Hertz, 1938; Lehrer, Horridge, Zhang, \& Gadagkar, 1995; Lunau, Wacht, \& Chittka, 1996; Menzel,
Ventura, Werner, Joaquim, \& Backhaus, 1989; Pessotti, 1967a; Pessotti, \& Lé Sénechal, 1981), mesmo quando os estímulos coloridos são apresentados em um monitor de computador (Brown, McKeon, Curley, Weston, Lambert, \& Lobowitz, 1998; Cooke, Couvillon, \& Bitterman, 2007).

Vários estudos também demonstraram a discriminação entre formas - padrões em preto e branco, ou figuras de formas diferentes mas com a mesma cor (e.g., Gould, 1985, 1986; Horridge, 2000, 2007; Lehrer, \& Campan, 2005; Lehrer et al., 1995; Moreno et al., 2012; Pessotti, \& Carli-Gomes, 1981; Rodriguez, Gumbert, de Ibarra, Hunze, \& Giurfa, 2004; Zhang, Srinivasan, \& Collett, 1995).

No estudo de Pessotti e Carli Gomes (1981) foram empregadas 30 abelhas da espécie Melipona quadrifasciata anthidioides, 28 abelhas da espécie Melipona rufiventris e 28 abelhas Apis mellifera adansonii em tarefas discriminativas com três diferentes tipos de estímulos. O objetivo era gerar uma classificação das abelhas quanto a aprendizagem. Foram comparados os resultados dos sujeitos em treinos de discriminação entre cores (azul e amarelo), entre presença ou ausência de lâmpada acesa e entre formas ("estrela" e "quadrado"). No treino de discriminação entre cores, foram utilizados como estímulos duas bandejas plásticas com diâmetro de $6 \mathrm{~cm}$, uma delas azul e a outra, amarela. No treino de discriminação simples entre formas, foram utilizadas placas de material plástico de cor azul celeste recortadas, uma delas, em forma de estrela de 8 pontas, com diâmetro de $4,5 \mathrm{~cm}$, e a outra, em forma de quadrado, com 4,5 $\mathrm{cm}$ de lado. Após 70 tentativas dessa tarefa, conduzidas com cada abelha, a porcentagem de acertos, em média, foi de 91,3\% para Melipona ruvifentris, 86,7\% para Melipona quadrifasciata anthidioides e 70,8\% para Apis melífera adansonii. As operárias da espécie Apis mellifera adansonii mostraram melhor desempenho, em relação aos sujeitos das duas outras espécies, nos treinos em que foram utilizadas as formas. A porcentagem de respostas discriminadas foi de $77 \%$. Para as abelhas da espécie Melipona rufiventris a porcentagem foi de $76 \%$ e para as abelhas da espécie Melipona quadrifasciata anthidioides a porcentagem foi de $75 \%$. Nos treinos em que foram utilizadas lâmpada acesa e lâmpada apagada como estímulos, o melhor desempenho foi obtido pelo grupo das Melipona quadrifasciata anthidioides: $78 \%$ de respostas discriminadas. O grupo das adansonii obteve $77 \%$ de acertos e o grupo das rufiventris obteve $75 \%$. De modo geral, quando se compara os estímulos, a discriminação de cores tendeu a ser melhor que a dos outros tipos de estímulos. É importante notar que nesse estudo, não foi usado um critério de estabilidade; a pergunta era o quanto as abelhas aprenderiam, dado o número pré-estabelecido de tentativas.

Em um experimento do estudo de Moreno et al. (2012), conduzido com abelhas da espécie Melipona rufiventris, oito sujeitos foram treinados individualmente a discriminar entre azul e amarelo e outros oito, entre dois padrões listrados em preto e branco (padrão horizontal 
versus padrão horizontal). $\mathrm{O}$ aparato usado no treino consistia em um túnel de madeira que terminava com duas saídas em Y. Nos dois braços terminais eram posicionados verticalmente os estímulos discriminativos. Cada estímulo era apresentado sobre uma placa quadrada com $12 \mathrm{~cm}$ de lado e com abertura circular no centro. Os sujeitos eram treinados a voar através do túnel e, em seguida, atravessar um dos estímulos discriminativos. O treino era organizado em blocos de 15 minutos, e o procedimento era encerrado ao ser atingido o critério de $100 \%$ de acertos em dois blocos consecutivos. A tarefa foi aprendida rapidamente, com os dois tipos de estímulos, por todas as abelhas, depois de menos duas horas de treino.

Abelhas podem também aprender a discriminar entre estímulos visuais compostos (Moreno, Varella, Canovas, Postalli, de Souza, \& Ventura, 2014) ou entre estímulos visuais consideravelmente mais complexos, incluindo representações de faces humanas (Bernard, Stach, \& Giurfa, 2006; Dyer, Neumeyer, \& Chittka, 2005), ou reproduções de pinturas (Chittka, \& Walker, 2007; Wu, Moreno, Tangen, \& Reinhard, 2013).

Além disso, vários estudos mostraram que abelhas podem aprender relações condicionais entre estímulos visuais (Cooke et al., 2007; Giurfa et al., 2001; Moreno et al., 2012) ou entre estímulos visuais e aromas (Giurfa et al., 2001; Reinhard, Srinivasan, \& Zhang, 2004; Srinivasan, Zhang, \& Zhu, 1998; Zhang, Lehrer, \& Srinivasan, 1999).

Nesse sentido, abelhas podem servir como um modelo animal para a investigação de condições para o estabelecimento do comportamento simbólico (ou "repertórios comportamentais pré-simbólicos", cf. Galvão, Soares Filho, Barros, \& Souza, 2008), ou mesmo como modelo animal para a investigação de fenômenos de importância clínica, como o responder sob controle restrito de estímulos (overselective stimulus control) (Moreno et al., 2012).

De especial interesse para a Análise do Comportamento são os estudos de Isaías Pessotti sobre aprendizagem em abelhas, conduzidos ainda a partir da década de 1960. Pessotti desenvolveu um programa completo para estudar o comportamento operante em abelhas, o qual envolvia modelagem de uma resposta instrumental, esquemas de reforçamento, punição e, principalmente, aprendizagem discriminativa (Pessotti, 1964, 1967a, 1967b, 1969, 1971, 1972, 1981; Pessotti \& Carli Gomes, 1981; Pessotti \& Lé Senechal, 1981; Pessotti, \& Otero, 1981)

Esse conjunto de estudos representa uma abordagem experimental alternativa aos métodos tipicamente empregados em outros laboratórios. Destacam-se os seguintes aspectos dessa abordagem: a) A resposta de escolha é definida como uma resposta operante (resposta de pressão à barra); b) Os sujeitos são treinados individualmente; c) Os dados são analisados individualmente, sendo representados por meio de curvas de frequência acumulada de respostas, o que permite uma análise refinada do desempenho de cada sujeito ao longo de todo o treino; d) $\mathrm{O}$ critério de aprendizagem pode ser definido como um determinado número de respostas corretas consecutivas, o que permite concluir seguramente se um determinado repertório foi estabelecido e se é estável. Em constraste, outros estudos (p.ex., Cooke et al., 2007; Giurfa et al., 2001; Horridge, 2000; Moreno et al., 2012; Reinhard et al., 2004) geralmente apresentam características como: a) A resposta de escolha é definida como simplesmente pousar sobre o estímulo ou voar através de um estímulo; b) Embora os sujeitos sejam individualmente marcados, os experimentos são conduzidos com vários sujeitos ao mesmo tempo; c) Os dados são agrupados, sendo representados em termos de porcentagens de acertos do grupo; d) Os critérios de aprendizagem são definidos com base em critérios de testes estatísticos, de tal modo que desempenhos que envolvem porcentagens de acertos em torno de, por exemplo, 65 a $70 \%$, são muitas vezes aceitos como demonstração de aprendizagem.

Um exemplo de abordagem experimental alternativa é o estudo de Pessotti (1969, 1981), em que o autor investigou o estabelecimento de relações condicionais entre estímulos visuais em Melipona rufiventris. Foram utilizados dois aparelhos iguais, posicionados lado a lado, e cobertos com discos de material plástico (ambos azuis ou ambos amarelos). No interior de cada aparelho estava localizada uma lâmpada. Cada uma das caixas possuía uma alavanca e um bebedouro. A resposta operante consistia em pressionar a alavanca, posicionada próxima ao bebedouro. Quando uma abelha pressionava a barra, essa resposta era automaticamente registrada por um sistema eletromecânico e operava um interruptor, produzindo a apresentação de uma solução de açúcar. A cada tentativa, cada um dos dois aparelhos era apresentado com um disco de uma cor (a cor era a mesma nos dois aparelhos) e esta cor era definida como estímulo modelo. Os estímulos de comparação eram constituídos por lâmpadas de 6 Watts: um dos aparelhos era apresentado com uma lâmpada acesa, o outro era apresentado com uma lâmpada apagada. Inicialmente Pessotti ensinava a cada um dos sujeitos a resposta de pressão à barra. Em seguida, cada sujeito era submetido a um treino de discriminação simples entre lâmpada acesa e lâmpada apagada. Quando era atingido o critério de seis acertos consecutivos, tinha início o treino de reversão de discriminação. Já no início dessa fase de reversão, um estímulo condicional era posicionado junto aos aparelhos: por balanceamento, para metade das abelhas os discos azuis eram apresentados em todas as tentativas dessa fase em que a lâmpada acesa funcionava como $\mathrm{S}+$ e a lâmpada apagada funcionava como S-. Após seis acertos consecutivos, tinha início a 2a. reversão de discriminação (quando a lâmpada apagada 
voltava a funcionar como $\mathrm{S}+$ ), com os discos amarelos sendo posicionados sobre os aparelhos em todas essas tentativas, até o início da próxima reversão. Esse processo de reversões sucessivas seguia até que fosse atingido o seguinte critério de aprendizagem: a) seis acertos consecutivos em uma fase de reversão; b) quatro acertos consecutivos a partir da primeira tentativa da reversão seguinte. Quando esse critério era atingido, tinha início a fase de treino de discriminação condicional propriamente dita, quando os estímulos condicionais se alternavam em uma sequência aleatória. O critério de aprendizagem da discriminação condicional, $75 \%$ de respostas corretas entre as 30 primeiras respostas dessa fase, foi atingido por todas as dez abelhas treinadas. $\mathrm{O}$ procedimento durava cinco dias (cinco sessões), em média, para cada abelha. Todas as dez abelhas atingiram o critério de aprendizagem. No entanto, deve-se destacar que nesse procedimento o autor empregou um procedimento em que o estímulo condicional e os estímulos de comparação eram apresentados simultaneamente. Essa característica do procedimento levanta a possibilidade de que os desempenhos observados resultaram de um responder sob controle de configurações de estímulos, em vez de um responder sob controle de relações entre os estímulos (Iversen, 1997; Iversen, Sidman, \& Carrigan, 1986; Kamil \& Sacks, 1972; Lionello \& Urcuioli, 1998). Contudo, o estudo de Pessotti é de especial interesse para um dos experimentos do presente estudo, o qual empregou aparelhos adaptados de seu estudo e investigou o estabelecimento de discriminações simples e reversões sucessivas de discriminação em Melipona quadrifasciata.

O objetivo do primeiro experimento do presente estudo foi estabelecer discriminações simples entre as cores azul e amarelo, entre preto e branco e entre padrões em preto e branco, ampliando-se a literatura sobre aprendizagem discriminativa em abelhas do gênero Melipona. O objetivo do segundo experimento foi estabelecer discriminação simples entre luzes azul e amarela e reversões sucessivas de discriminação, usandose um equipamento automatizado, adaptado de Pessotti $(1969,1981)$ e similar ao usado no estudo de Moreno et al. (2014).

\section{EXPERIMENTO 1- DISCRIMINAÇÃO SIMPLES ENTRE CORES E ENTRE PADRÕES EM PRETO E BRANCO}

\section{Sujeitos}

\section{MÉTODO}

Foram empregadas 28 abelhas da espécie Melipona quadrifasciata, operárias, em idade adulta, experimentalmente ingênuas, todas procedentes de uma mesma colmeia instalada no Laboratório de Psicologia da Aprendizagem do Departamento de Psicologia da Universidade Federal de São Carlos.

\section{Equipamentos e materiais}

Foram utilizados dois aparelhos experimentais simultaneamente. Cada aparelho é uma vasilha metálica de fundo retangular medindo $12,6 \mathrm{~cm} \mathrm{X} \mathrm{7,0} \mathrm{cm} \mathrm{e} 3,5 \mathrm{~cm}$ de altura, coberta por uma tampa metálica (Figura 1). A tampa contém um conjunto de cinco orifícios (bebedouro) através dos quais a abelha pode sugar uma solução de açúcar a $75 \%$ (xarope). Uma pequena concha contendo xarope pode ser aproximada ao bebedouro, ou mantida distante dele, por uma haste operada manualmente pelo experimentador. Os dois aparelhos eram dispostos sobre uma mesa de tampo branco, a 1,5 m da colmeia, com uma distância de $35 \mathrm{~cm}$ entre eles. A cada tentativa um dos aparelhos portava o $\mathrm{S}+\mathrm{e}$ o outro portava o $\mathrm{S}$ -

Os estímulos discriminativos eram superfícies de mesma cor (azul, amarelo, branco ou preto), apresentadas em placas quadradas com $7 \mathrm{~cm}$ de lado, feitas de material emborrachado (E.V.A.). Também foram utilizados como estímulos discriminativos diferentes padrões em preto e branco, os quais consistiam em figuras pretas recortadas em material adesivo, em material do tipo contact, e afixadas em quadrados de material emborrachado de cor branca.

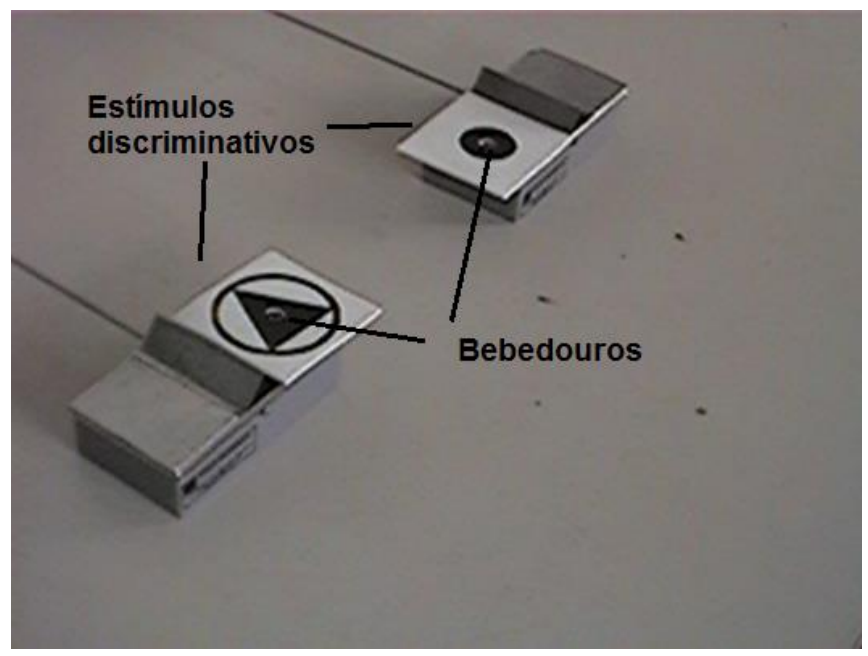

Figura 1. Situação experimental durante treino de discriminação simples entre dois estímulos visuais (padrões em preto e branco). Em uma tentativa de treino, uma resposta correta era definida como pousar sobre o aparelho sinalizado com S+. Essa resposta era seguida de acesso ao bebedouro. Após uma resposta incorreta (ao S-), o acesso ao bebedouro era bloqueado.

\section{Procedimento}

Cada abelha era individualmente treinada em uma sessão com duração de aproximadamente oito horas.

\section{a) Identificação do sujeito.}

Cada abelha recebia uma marca sobre o dorso feita com tinta guache (cf. Pessotti, 1964). A marca era feita durante a resposta de sugar no bebedouro, na fase inicial do procedimento. Quando encontra o xarope, a abelha 
suga por aproximadamente $30 \mathrm{~s}$, tempo suficiente para marcar o dorso com um pincel.

\section{b) Modelagem de pouso sobre o aparelho e modelagem da resposta de sugar no bebedouro \\ Um pequeno recipiente com xarope era} inicialmente colocado à porta da colmeia, e então era gradualmente deslocado em direção aos aparelhos experimentais. Em seguida, algumas gotas de xarope eram depositadas sobre a tampa de um dos aparelhos experimentais.

Quando a abelha marcada estivesse regularmente recolhendo xarope nessa área, algumas gotas de xarope passavam a ser depositadas próximo ao bebedouro de um dos aparelhos experimentais (o outro aparelho permanecia coberto). Em seguida, a tampa era limpa e o xarope ficava disponível apenas no bebedouro. Quando a abelha aprendia a recolher xarope consistentemente em um dos aparelhos, esse era tampado e o outro ficava disponível. Então, o mesmo procedimento era repetido nesse segundo aparelho.

\section{c) Discriminação simples.}

Em cada tentativa de treino, o estímulo definido como S+ e o estímulo definido como S- eram simultaneamente posicionados, sobre cada um dos dois os aparelhos experimentais. A posição dos estímulos (lado "direito" ou lado "esquerdo") era alternada entre as tentativas, em uma sequência pseudo-randômica definida previamente em um protocolo de treino.

Cada resposta de pouso sobre o estímulo definido como correto dava acesso ao xarope ad libitum. As respostas corretas eram, portanto, reforçadas em um esquema de reforçamento contínuo. Após recolher o xarope, a abelha retornava à colmeia e, tipicamente, voava de volta ao aparato experimental cerca de um a dois minutos mais tarde, o que definia o início de uma nova tentativa (ou visita).

Uma resposta de pousar sobre o estímulo definido como S- era definida como incorreta e não tinha consequência programada. $\mathrm{O}$ alimento desse aparelho era mantido inacessível. Nessa situação, era permitido que a abelha voasse até o aparelho sinalizado com $\mathrm{S}+$ e sugasse o xarope, mas apenas a primeira resposta (incorreta) era registrada.

Como ilustrado na Figura 2, duas abelhas foram treinadas a discriminar entre azul e amarelo. Duas outras abelhas foram treinadas a discriminar entre preto e branco. Vinte abelhas foram treinadas a discriminar entre dois padrões em preto e branco.

$\mathrm{O}$ procedimento completo era encerrado ao ser atingido o critério de aprendizagem de dez respostas corretas consecutivas ou ao final de uma sessão sem aprendizagem. Tipicamente, uma sessão podia apresentar em torno de 150 tentativas. Uma medida de aprendizagem complementar - o índice de discriminação - foi adotada considerando-se a porcentagem de respostas corretas para as dez últimas tentativas de treino.

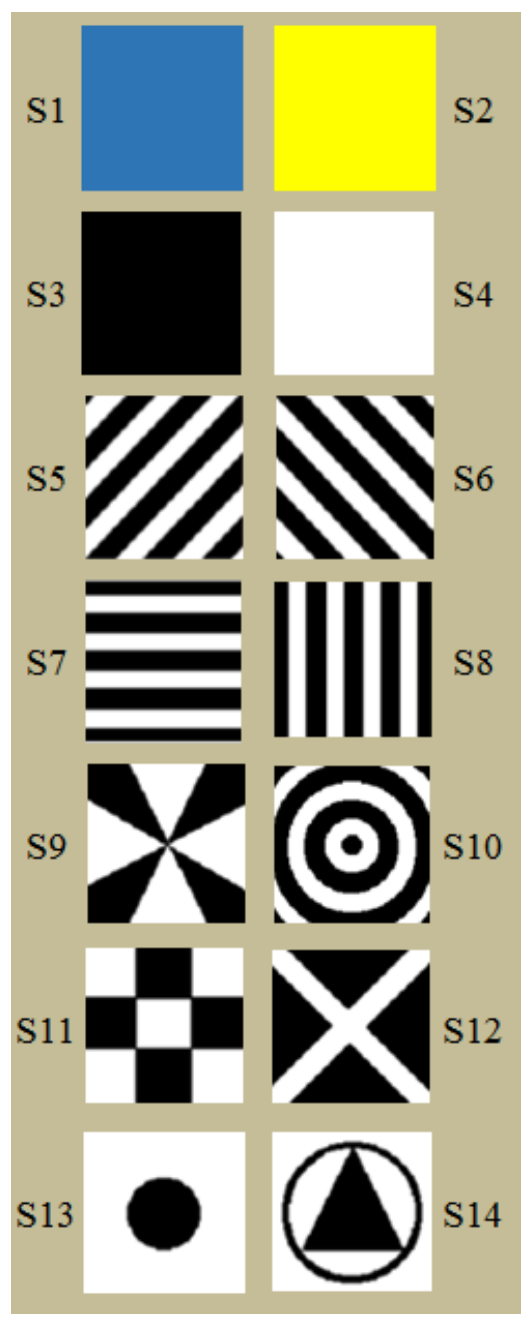

Figura 2. Conjunto de estímulos utilizados para treino de discriminação simples no Experimento 1. Cada um desses pares de estímulos foi utilizado com quatro abelhas. Os estímulos S5, S6, S7 e S8 são descritos, por exemplo, por Giurfa et al. (2001). O estímulo S9 é uma adaptação do estudo de Gould (1996). Os estímulos S11, S12, S13 e S14 são adaptações de estímulos usados por Galvão (1993).

\section{RESULTADOS}

A Figura 3 apresenta a frequência acumulada de respostas para todas as abelhas utilizadas no experimento. Nestes gráficos, a presença ou ausência de discriminação é observada por diferenças de aceleração das curvas de respostas corretas (linhas pretas) e de curvas de respostas incorretas (linhas cinzas). De acordo com o critério de aprendizagem, quando a discriminação é estabelecida, a frequência de respostas corretas aumenta continuamente (aceleração constante ou positiva), enquanto a curva de respostas incorretas torna-se negativamente acelerada, registrando a ausência de respostas ao $\mathrm{S}$ - nas dez tentativas finais. 

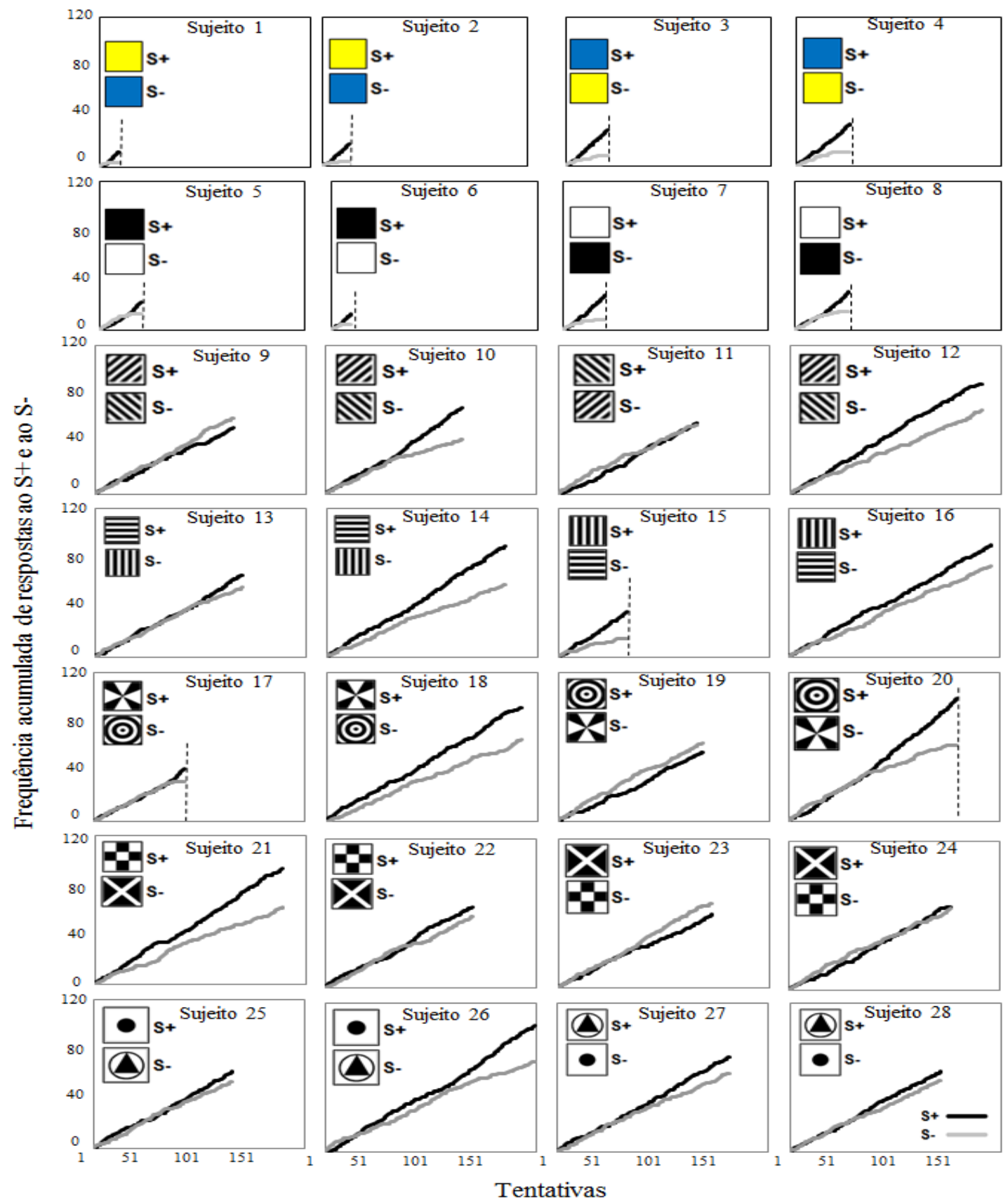

Figura 3. Frequência acumulada de respostas corretas (ao S+) e incorretas (ao S-) para abelhas individuais durante treino de discriminação entre estímulos visuais (azul, amarelo, preto, branco e padrões em preto e branco). Linhas escuras representam frequência acumulada de respostas ao S+; linhas cinzas representam frequência acumulada de respostas ao S-. Linhas verticais tracejadas indicam o momento do treino em que foi atingido o critério de 10 acertos consecutivos.

Os quatro primeiros gráficos referem-se ao treino de discriminação simples entre as cores amarelo e azul (S1 versus $\mathrm{S} 2$ ) com quatro abelhas. Todas as quatro atingiram o critério de aprendizagem. O número de visitas até que o critério de dez acertos consecutivos fosse atingido variou entre 18 e 51 visitas.
O terceiro conjunto de gráficos representa os resultados de abelhas que receberam treino de discriminação entre preto e branco (S3 versus $\mathrm{S} 4$ ). Todas as quatro abelhas dessa condição também aprenderam a tarefa. O número de respostas requeridas para se atingir o critério de dez acertos consecutivos variou entre 20 e 52 visitas. 
A próxima sequência de gráficos apresenta os resultados da discriminação entre "padrões inclinados" (S5 versus S6). Nessa tarefa, nenhuma das quatro abelhas atingiu o critério de aprendizagem. Para duas abelhas, S10 e S12, a curva acumulada de respostas discriminadas se destacou da curva acumulada de respostas incorretas em alguns momentos do treino. Contudo, o índice de discriminação para $\mathrm{S} 10$ e $\mathrm{S} 12$, considerando-se as dez últimas tentativas, foi, respectivamente, de $60 \%$ e $40 \%$.

A quarta linha da Figura 3 apresenta os resultados do treino de discriminação entre padrões verticais e padrões horizontais (S7 versus S8). Com esse par, uma dentre quatro abelhas (Sujeito 15) atingiu o critério de dez acertos consecutivos. O índice de discriminação para as demais abelhas nas dez tentativas finais variou entre $40 \%$ (S13) e 60\% (S14 e S16).

A quinta linha da Figura 3 apresenta os resultados do treino de discriminação entre "padrão radiais" e "padrões circulares" (S9 versus S10). Duas abelhas (S17 e S20) atingiram o critério de aprendizagem. O Sujeito 17 atingiu o critério após 85 tentativas e o Sujeito 20 após 160 tentativas. Para S18, a curva acumulada de respostas discriminadas se destacou da curva acumulada de respostas incorretas aproximadamente depois 100 tentativas. Contudo, o índice de discriminação para essa abelha foi de $40 \%$ nas últimas dez tentativas.

Para o penúltimo par de estímulos, nenhuma abelha atingiu o critério de aprendizagem. A abelha S21 apresentou uma proporção de respostas corretas destacadamente superior à proporção de respostas incorretas, mas o índice de discriminação foi de $60 \%$ considerando-se as dez tentativas finais.

Para o último par de estímulos, do mesmo modo, nenhuma abelha atingiu o critério de aprendizagem. O melhor desempenho foi apresentado pela abelha S26, que embora não tenha atingido o critério, apresentou índice de discriminação de $70 \%$ entre as últimas dez tentativas.

\section{EXPERIMENTO 2 - DISCRIMINAÇÕES SIMPLES E REVERSÕES DE DISCRIMINAÇÃO COM RESPOSTA OPERANTE EM APARATO AUTOMATIZADO}

\section{Sujeitos}

\section{MÉTODO}

Foram empregadas quatro abelhas da espécie Melipona quadrifasciata, operárias, em idade adulta, experimentalmente ingênuas, todas procedentes de uma mesma colmeia instalada no Laboratório de Psicologia da Aprendizagem do Departamento de Psicologia da Universidade Federal de São Carlos.

Cada um dos dois aparelhos automáticos utilizado neste experimento era formado por uma caixa de acrílico preto, com fundo retangular medindo $15 \mathrm{~cm} \mathrm{X} \mathrm{7,0} \mathrm{cm} \mathrm{e} \mathrm{4,5}$ $\mathrm{cm}$ de altura (Figura 4). No interior de cada aparelho havia um recipiente contendo xarope e um mecanismo que elevava uma concha com xarope até o bebedouro. $\mathrm{Na}$ área externa do aparelho existia uma alavanca, distante cerca de $1,5 \mathrm{~cm}$ do bebedouro. Na parte posterior do aparelho eram encerradas duas lâmpadas incandescentes, de $4 \mathrm{~W}$, visíveis através de uma janela de acrílico transparente. $\mathrm{O}$ aparato permitia projetar em cada aparelho uma luz azul ou uma luz amarela, as quais funcionavam como estímulos discriminativos.

$\mathrm{O}$ aparato era controlado eletronicamente, por meio de uma interface ligada a um microcomputador e um software desenvolvido para este estudo. Os dois aparelhos eram dispostos sobre uma mesa de tampo branco posicionada a uma distância de aproximadamente $1,5 \mathrm{~m}$ da colmeia. A distância entre os dois aparelhos era de $35 \mathrm{~cm}$.

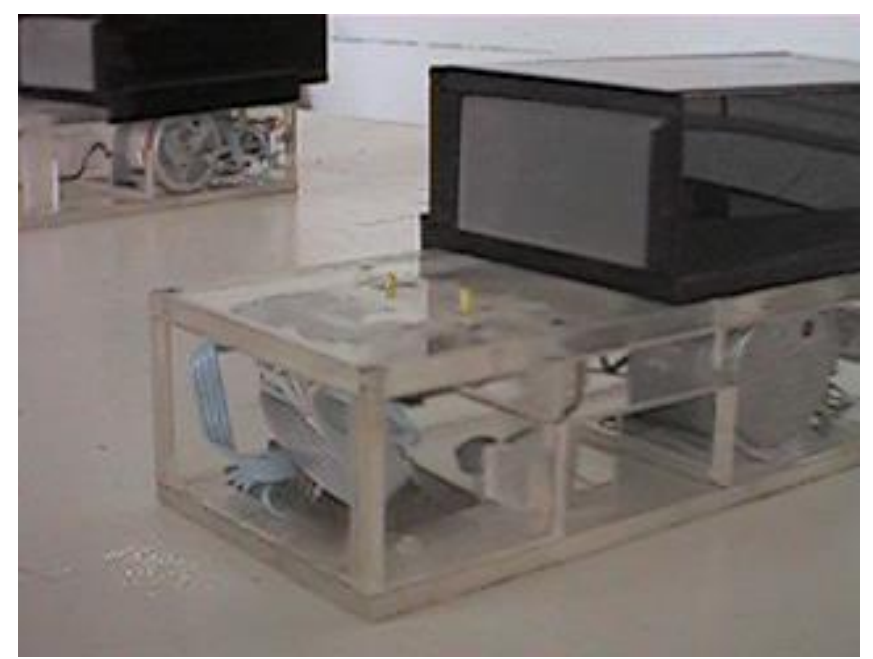

Figura 4. Situação experimental durante treino de discriminação simples e reversões de discriminação no Experimento 2. Foram usados aparelhos com controle automático; toda a contingência de reforçamento era controlada por uma interface ligada a um computador. No centro da área de pouso (superfície transparente à frente dos aparelhos) encontram-se duas alavancas verticais para resposta operante (barras verticais amarelas). Na parte de trás de cada aparelho encontra-se a caixa (de acrílico preto) com duas lâmpadas (na foto, desligadas), utilizadas para projetar os estímulos discriminativos.

\section{Procedimento \\ a) Identificação do sujeito}

Cada abelha recebeu uma marca sobre o dorso feita com tinta guache (cf. Pessotti, 1964).

\section{b) Modelagem de pouso sobre o aparelho}

A modelagem de pouso seguiu o mesmo procedimento do Experimento 1 .

\section{c) Modelagem de pressão à alavanca}

O xarope passava a ser disponível apenas com a aproximação do sujeito à alavanca. Posteriormente, o sujeito recebia reforço apenas quando tocasse a alavanca. Finalmente, apenas as respostas de pressão à alavanca eram reforçadas. Após a primeira pressão à alavanca, o segundo aparelho era colocado sobre a mesa e o sujeito era treinado a pressionar a alavanca nesse outro aparelho. Após dez visitas em que fossem registradas pressões à alavanca em um dos aparelhos, iniciava-se a fase de discriminação simples. 


\section{d) Discriminação simples}

Uma tentativa de treino iniciava com a apresentação dos estímulos discriminativos nos aparelhos (luz azul em um aparelho; luz amarela em outro).

Uma resposta de pressão à barra no aparelho sinalizado com $\mathrm{S}+$ era seguida de acesso ao bebedouro por $30 \mathrm{~s}$.

Uma resposta de pressão à barra no aparelho sinalizado com S- era seguida de um período de $4 \mathrm{~s}$ em que as luzes dos aparelhos eram mantidas desligadas e $\mathrm{o}$ bebedouro era mantido bloqueado.

A posição do $\mathrm{S}+$ e do $\mathrm{S}$ - era variada ao longo das tentativas; os estímulos empregados como $\mathrm{S}+$ e S- foram contrabalanceados entre as abelhas (ver Figura 5).

O critério de encerramento do treino de discriminação era de dez respostas corretas consecutivas.

\section{e) Reversões de discriminação}

As funções dos estímulos de comparação (S+e S-) eram revertidas. $O$ treino na primeira reversão era encerrado após dez respostas corretas consecutivas. A segunda e a terceira reversões eram encerradas após seis respostas corretas consecutivas. A reversões subsequentes eram encerradas após atingido o critério de três respostas corretas consecutivas. O critério de encerramento da série de reversões era de duas reversões consecutivas sem erros.

\section{RESULTADOS}

A Figura 5 apresenta curvas de frequência acumulada de respostas ao S+ e ao S- durante treino inicial de discriminação simples e as sucessivas reversões para os Sujeitos 62, 63, 64 e 65. As linhas escuras indicam curvas acumuladas de respostas ao $\mathrm{S}+$; as linhas claras indicam curvas de respostas ao S-. Linhas verticais tracejadas indicam que o critério de aprendizagem foi atingido e seguido por uma reversão.

$\mathrm{Na}$ fase de discriminação simples, o Sujeito 62 (S+ azul) atingiu o critério de dez respostas corretas consecutivas após 31 respostas. Na primeira reversão de discriminação, o critério foi atingido após 25 respostas. Na segunda e terceira reversões, em que o critério de aprendizagem era de seis respostas discriminadas consecutivas, o sujeito atingiu esse critério após 29 e 19 respostas, respectivamente. Na quarta reversão o critério de aprendizagem era de três acertos consecutivos e foi atingido com 18 visitas. Com o início da quinta reversão, o sujeito apresentou três respostas corretas consecutivas, mas abandonou a sessão experimental em seguida.

O Sujeito 63 atingiu o critério de aprendizagem na fase inicial de discriminação (S+ amarelo) após 87 visitas. Na primeira reversão o critério foi atingido após 225 visitas. Nessa fase foram observadas longas pausas entre as visitas. $\mathrm{Na}$ fase seguinte, após 37 visitas, o sujeito abandonou definitivamente a sessão experimental.

Para o Sujeito 64 o critério de aprendizagem (S+ azul) foi atingido com 23 respostas. A primeira reversão foi concluída após 148 respostas. O número de respostas necessárias para se atingir o critério de aprendizagem nas quatro fases subsequentes foi $65,42,28$ e 28 , respectivamente. Durante essas fases de reversão, em que longas pausas entre as visitas tornaram-se frequentes, foi observada a deterioração da resposta operante. Foram realizadas outras sete fases de reversão de discriminação, nas quais o critério de aprendizagem era sempre de três respostas discriminadas consecutivas. Durante essa fase, não foi observada a regularização da resposta operante. Em seguida, o sujeito abandonou definitivamente a sessão experimental.

O Sujeito 65 atingiu o critério de aprendizagem com 76 respostas e nas reversões o critério foi atingido após 94, 24 e 19 respostas. Após cinco novas reversões (em 260 tentativas), o sujeito abandonou a sessão experimental.

\section{DISCUSSÃO}

Os resultados do Experimento 1 mostraram que abelhas da espécie Melipona quadrifasciata aprenderam rapidamente a discriminar entre azul e amarelo ou entre preto e branco. Com as abelhas que discriminaram entre os estímulos amarelo e azul, foram necessárias, em média, 35 visitas para completar a tarefa, o que confirma os dados da literatura com estas cores (e.g., Moreno et al., 2012; Pessotti, 1967; Pessotti \& Carli-Gomes, 1981). Resultados similares foram encontrados na tarefa de discriminação entre preto e branco. Por outro lado, apenas três abelhas aprenderam a tarefa de discriminar entre dois padrões em preto e branco. Duas abelhas atingiram o critério de aprendizagem na discriminação entre os estímulos "padrão radial" e "padrão circular", enquanto uma abelha atingiu o critério na discriminação entre "padrão vertical" e "padrão horizontal". Esses resultados contrastam com os resultados de Moreno et al. (2012) e de Pessotti e Carli-Gomes (1981), que demonstraram rápida aprendizagem de discriminação entre formas. Uma diferença entre o procedimento do Experimento 1 e o procedimento do estudo de Pessotti e Carli-Gomes diz respeito às cores utilizadas na apresentação das figuras, embora, em ambos os casos, a aquisição da tarefa dependesse necessariamente da discriminação de formas (não poderia se basear somente em propriedades cromáticas). No presente estudo as figuras eram pretas sobre fundo branco, enquanto no estudo de Pessotti e Carli-Gomes as formas (uma estrela de oito pontas versus um quadrado) eram (ambas) figuras recortadas em material azul. Contudo, não existem dados na literatura que sugiram que a discriminação de formas seja mais facilmente estabelecida a depender das cores utilizadas.

Pode ser sugerido que essa diferença nos resultados se deve ao fato de que no presente experimento foram utilizadas abelhas da espécie Melipona quadrifasciata, enquanto o estudo de Moreno et al. (2012) empregou Melipona rufiventris. Contudo, o estudo de Pessotti e Carli-Gomes (1981) sugere que essas duas espécies de melíponas apresentam desempenho semelhante em uma tarefa de discriminação entre formas. 


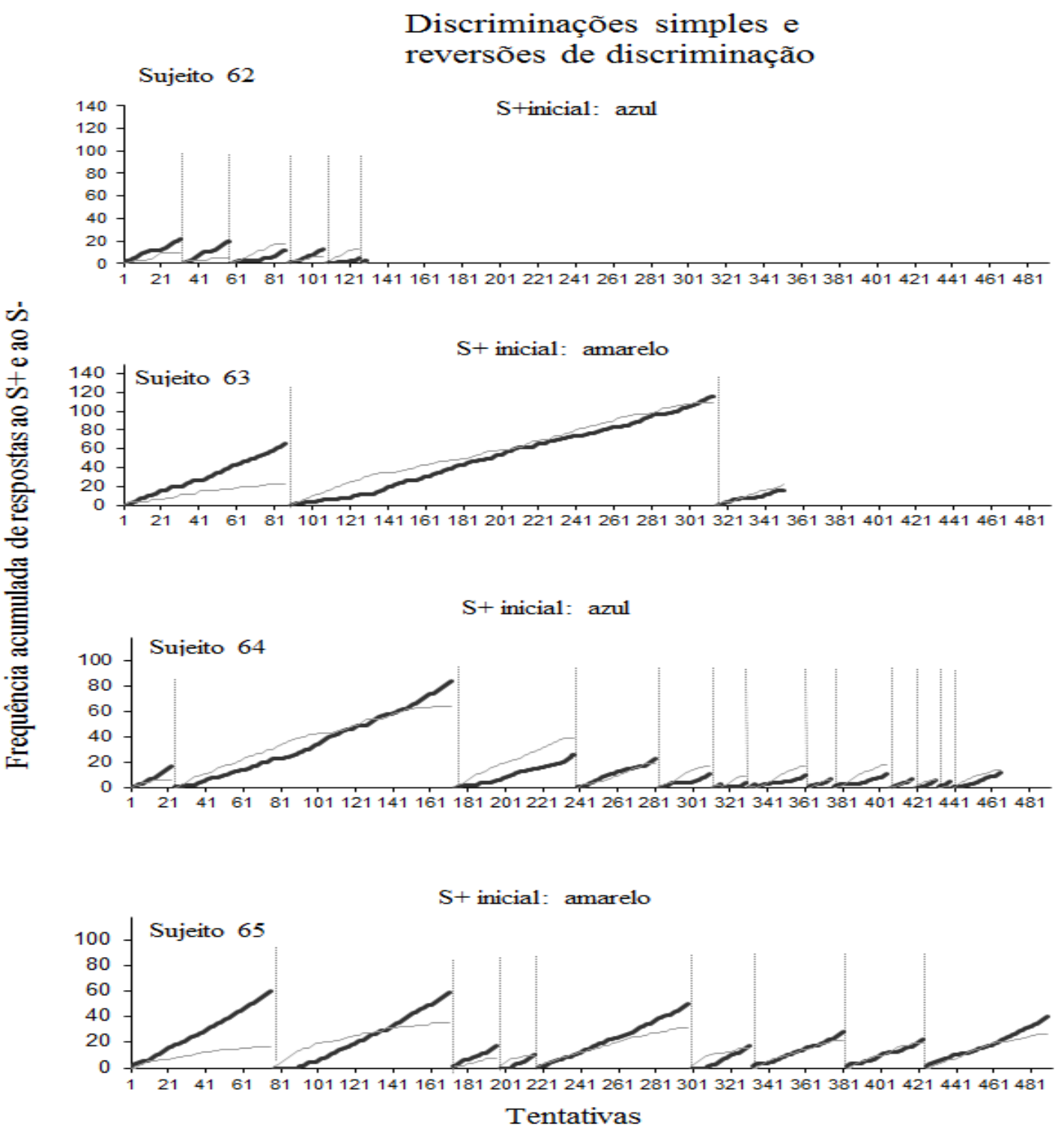

Figura 5. Frequência acumulada de respostas durante treino de discriminação simples e reversões sucessivas com quatro melíponas. Os estímulos discriminativos eram luz azul e luz amarela, projetados atrás das barras (Figura 4). As linhas escuras indicam respostas ao S+. As linhas claras indicam respostas ao S-. As linhas verticais tracejadas separam as fases de reversão. O critério de aquisição e para a primeira reversão era de 10 respostas corretas consecutivas. O critério para a $2 \mathrm{a}$. e $3 \mathrm{a}$. reversões era de seis respostas corretas consecutivas. O critério para reversões subsequentes era de três respostas corretas consecutivas.

Uma hipótese mais plausível para explicar os dados negativos obtidos nas tarefas de discriminação entre padrões no Experimento 1 diz respeito às posições dos estímulos, que eram dispostos horizontalmente sobre os aparelhos experimentais e os sujeitos pousavam nas bordas dos estímulos. No estudo de Moreno et al. (2012), por outro lado, os estímulos eram posicionados verticalmente e os sujeitos voavam através de uma abertura no centro dos estímulos. De fato, esse aspecto de procedimento está presente em grande parte dos estudos sobre aprendizagem discriminativa em abelhas (p.ex., Giurfa et al., 2001; Horridge, 2000; Reinhard, Srinivasan, \& Zhang, 2004; Wu, Moreno, Tangen, \& Reinhard, 2013). Estudos futuros poderiam replicar o Experimento 1 usando os mesmos pares de estímulos, porém usando um aparato em que os estímulos fossem apresentados verticalmente, o que facilitaria a comparação dos dados produzidos com os dados de uma ampla literatura.

Quanto ao Estudo 2, um dos resultados mais importantes é a confirmação da viabilidade do emprego de um procedimento de treino discriminativo automatizado com abelhas (Moreno et al., 2012; Pessotti, 1969, 1981). Nos estudos de Pessotti (1969) e de Moreno et al. (2012), o registro da resposta operante e a apresentação do estímulo reforçador eram automatizados, mas a apresentação dos estímulos discriminativos era controlada, ao menos em parte, 
pelo experimentador. No presente estudo, contudo, todo o procedimento era automatizado.

Os resultados do Experimento 2 também corroboram os dados de Pessotti $(1969,1981)$ quanto à possibilidade de se estabelecer em abelhas uma série de reversões sucessivas de discriminação. Esse tipo de desempenho é especialmente importante em tarefas que envolvem o estabelecimento de repertórios mais complexos, como discriminações condicionais, os quais requerem reversões rápidas de discriminação. Entre as três abelhas com melhor desempenho, foram estabelecidas entre quatro e 11 reversões de discriminação. $\mathrm{O}$ pior desempenho foi apresentado por uma abelha que aprendeu apenas uma reversão de discriminação. Durante a primeira reversão de discriminação, dois sujeitos apresentaram mais de 130 respostas até atingir o critério. Entretanto, esses resultados devem ser interpretados com cautela, uma vez que dificuldades experimentais, como falhas no equipamento, podem ter influenciado a aprendizagem dos sujeitos. Ao longo do procedimento, observou-se deterioração da resposta operante em vários momentos. Provavelmente por essa razão, foram registradas várias pausas no responder ao longo do treino com todos os sujeitos. Ao menos para alguns sujeitos, essa dificuldade em se estabelecer a resposta operante deve ter determinado a deterioração da aprendizagem discriminativa.

Os desempenhos das quatro abelhas do Experimento 2 podem ser considerados bastante inferiores aos desempenhos das dez abelhas do estudo de Pessotti (1967, 1981). Naquele estudo, as reversões de discriminação foram estabelecidas de modo rápido e estável com todas as abelhas. Contudo, um aspecto do Experimento 2 a ser destacado é o uso de lâmpadas coloridas como estímulos discriminativos. Treinos discriminativos no estudo de Pessotti $(1967,1981)$ envolviam discriminar entre lâmpada acesa e lâmpada apagada, mas não discriminar entre luzes de diferentes cores. Por um lado, o presente estudo sugere que as luzes coloridas utilizadas podem simular as cores de flores naturais. Por outro lado, esses resultados sugerem que as luzes utilizadas no Experimento 2 não possuem propriedades físicas que "imitem" flores naturais tão bem quanto os estímulos coloridos formados por materiais como E.V.A., plástico e papel. Por exemplo, duas abelhas adquiriram a primeira tarefa de discriminação apenas depois de cerca de 80 tentativas de treino, um desempenho nitidamente inferior ao desempenho das abelhas do Experimento 1, também treinadas a discriminar entre azul e amarelo, mas com estímulos formados por material emborrachado.

Em conjunto, os resultados de ambos os experimentos confirmam que melíponas podem aprender tarefas discriminativas, e a discriminação pode ser estabelecida tanto com a resposta de pousar sobre o estímulo $\mathrm{S}+\mathrm{e}$ inserir a glossa no bebedouro (no equipamento controlado pelo experimentador no Experimento 1), quanto com a resposta de pressão à barra (no equipamento com controle automatizado no Experimento 2). O uso do procedimento de treino discriminativo automatizado com abelhas pode facilitar o controle experimental, permitindo análises mais refinadas do desempenho de abelhas individuais em tarefas de aprendizagem operante; uma possibilidade a ser explorada, é a de colocar a resposta de pressão à barra sob controle de esquemas, aumentando assim a amostra de respostas a cada visita (em vez de uma única resposta, como nos dois experimentos relatados).

Os resultados de ambos os experimentos também mostraram que os desempenhos podem variar consideravelmente a depender dos estímulos discriminativos utilizados. O comportamento discriminativo de melíponas (abelhas das américas) tem sido muito menos explorado do que as de outras abelhas encontradas principalmente no continente europeu (cf. Moreno et al., 2012), o que justifica a continuidade de investigações visando desenvolver procedimentos que reduzam a variabilidade comportamental descrita por meio do controle rigoroso de variáveis (tanto no que concerne aos estímulos, quanto às respostas e aos esquemas de consequenciação). Um bom modelo experimental para investigar a aprendizagem discriminativa destas espécies de abelhas pode contribuir para seu uso como modelo animal para processos comportamentais básicos e para a investigação de outras variáveis e outros processos, como por exemplo, os efeitos de fármacos sobre o comportamento operante e sobre a aprendizagem.

\section{REFERÊNCIAS}

Backhaus, W. (1993). Color vision and color choice behavior of the honey bee. Apidologie, 24, 309-331.

Benard, J., Stach, S., \& Giurfa, M. (2006). Categorization of visual stimuli in the honeybee Apis mellifera. Animal Cognition, 9, 257-270.

Brown, M.F., McKeon, D., Curley, T., Weston, B., Lambert, C., \& Lebowitz, B. (1998). Working memory for color in honeybees. Animal Learning \& Behavior, 26(3), 264271.

Burton, A.M., Bruce, V., \& Hancock, P.J.B. (1999). From pixels to people: A model of familiar face recognition. Cognitive Science, 23, 1-31.

Chittka, L., \& Walker, J. (2007). Insects as art lovers: Bees for Van Gogh. Antennae, 2, 37-42.

Cooke, M.H., Couvillon, P.A., \& Bitterman, M.E. (2007). Delayed symbolic matching in honeybees (Apis mellifera). Journal of Comparative Psychology, 121(1), 106-108.

Dyer, A.G., \& Chittka, L. (2004). Biological significance of discriminating between similar colours in spectrally variable illumination: Bumblebees as a study case. Journal of Comparative Physiology A, 190, 105-114.

Dyer, A.G., Neumeyer, C., \& Chittka, L. (2005). Honeybee (Apis mellifera) vision can discriminate between and recognise images of human faces. Journal of Experimental Biology, 208, 4709-4714. 
Galvão, O.F. (1993). Classes funcionais e equivalência de estímulos. Psicologia: Teoria e Pesquisa, 9(3), 547-554.

Galvão, O.F., Soares Filho, P.S.D., Barros, R.S., \& Souza, C.B.A. (2008). Matching-to-sample as a model of symbolic behavior for bio-behavioral investigations. Reviews in Neurosciences, 19, 149-156.

Giurfa, M., Núñez, J., Chittka, L., \& Menzel, R. (1995). Colour preferences of flower-naïve honeybees. Journal of Comparative Physiology A, 177, 247-259.

Giurfa, M., Vorobyev, M., Kevan, P., \& Menzel, R. (1996). Detection of coloured stimuli by honeybees: Minimum visual angles and receptor specific contrasts. Journal of Comparative Physiology A, 178, 699-709.

Giurfa, M., Zhang, S., Jenett, A., Menzel, R., \& Srinivasan, M.V. (2001). The concepts of 'sameness' and 'difference' in an insect. Nature, 410, 930-932.

Gould, J.L. (1985). How bees remember flower shapes. Science, 227, 1492-1494.

Gould, J.L. (1986). Pattern learning by honeybees. Animal Behavior, 34, 990-997.

Gould, J.L. (1996). Specializations in honey bee learning. In C.F. Moss, \& S.J. Shettleworth (Eds.), Neuroethological studies of cognitive and perceptual processes (pp. 1129). Boulder: Westview Press.

Gumbert, A. (2000). Color choices by bumble bees (Bombus terrestris): Innate preferences and generalization after learning. Behavioral Ecology and Sociobiology, 48, 3643.

Hempel de Ibarra, N., Giurfa, M., \& Vorobyev, M. (2002). Discrimination of coloured patterns by honeybees through chromatic and achromatic cues. Journal of Comparative Physiology A, 188, 503-512.

Hertz, M. (1929). Figural perception in bees. Em W. D. Ellis (Ed.) A sourcebook of Gestalt Psychology (pp. 253-263). London: Kegan Paul, Trench, Trubner and Co. DOI: 10.1037/11496-021

Horridge, A. (2000). Seven experiments on pattern vision of the honeybee, with a model. Vision Research, 40, 25892603.

Horridge, A. (2007). The preferences of the honeybee (Apis mellifera) for different visual cues during the learning process. Journal of Insect Physiology, 53, 877-889.

Iversen, I.H. (1997). Matching-to-sample performance in rats: A case of mistaken identity? Journal of the Experimental Analysis of Behavior, 68, 27-47.

Iversen, I.H., Sidman, M., \& Carrigan, P. (1986). Stimulus definition in conditional discriminations. Journal of the Experimental Analysis of Behavior, 45, 297-304.

Kamil, A.C., \& Sacks, R.A. (1972). Three-configuration matching-to-sample in the pigeons. Journal of the Experimental Analysis of Behavior, 17, 483-488.

Lehrer, M, \& Campan, R. (2005). Generalization of convex shapes by bees: What are shapes made of? Journal of Experimental Biology, 208, 3233-3247.

Lehrer, M., Horridge, G.A., Zhang, S.W., \& Gadagkar, R. (1995). Shape vision in bees: Innate preference for flower-like patterns. Philosophical Transactions of the Royal Society of London, 347, 123-137.

Lionello, K.M., \& Urcuioli, P.J. (1998). Control by sample location in pigeons' matching to sample. Journal of the Experimental Analysis of Behavior, 70, 235-251.

Lunau, K., Wacht, S., \& Chittka, L. (1996). Colour choices of naive bumble bees and their implications for colour perception. Journal of Comparative Physiology A, 178, 477-489.

Menzel, R., Ventura, D. F., Werner, A., Joaquim, L. C. M., \& Backhaus, W. (1989). Spectral sensitivity of single photoreceptors and color-vision in the stingless bee, Melipona quadrifasciata. Journal of Comparative Physiology A- Sensory Neural and Behavioral Physiology, 166(2), 151-164.

Moreno, A.M., de Souza, D.G., \& Reinhard, J. (2012). A comparative study of relational learning capacity in honeybees (A. mellifera) and stingless bees ( $M$. rufiventris). PLoS One, 7(12). e51467.

Moreno, A.M., Varella, A.A.B., Canovas, D.S., Postalli, L.M.M., Ventura, D.F., \& de Souza, D.G. (2014). Assessing restricted stimulus control in typically developing preschool children and bees (Melipona quadrifasciata). Psychology \& Neuroscience, 7(2), 207220. DOI: $10.3922 /$ j.psns.2014.12

Pessotti, I. (1964). Estudo sobre aprendizagem e extinção em Apis mellifera. Jornal Brasileiro de Psicologia, 1(2), 97100.

Pessotti, I. (1967a). Aprendizagem de discriminação como um critério de classificação de abelhas. Revista Interamericana de Psicologia, 1(3), 177-187.

Pessotti, I. (1967b). Inversões de discriminação com dois "manipulanda" em Melipona (M) rufiventris. Revista de Psicologia Normal e Patológica, 3-4, 171-182.

Pessotti, I. (1969). Discriminação em Melipona (Micherenia) rufiventris Lepertier. Tese de Doutorado. Departamento de Psicologia Social e Experimental da Faculdade de Filosofia, Ciências e Letras da Universidade de São Paulo. São Paulo.

Pessotti, I. (1972). Discrimination with light stimuli and a lever-pressing response in Melipona rufiventris. Journal of Apicultural Research, 11(2), 89-93.

Pessotti, I. (1981). Aprendizagem em abelhas. VI Discriminação condicional em Melipona rufiventris. Revista Brasileira de Psicologia, 41(4), 681-693.

Pessotti, I., \& Carli-Gomes, M.J. (1981). Aprendizagem em abelhas. III: Discriminação com três tipos de estímulos visuais. Revista Brasileira de Biologia, 41(3), 667-672.

Pessotti, I., \& Lé Senechal, A.M. (1981). Aprendizagem em abelhas. I - Discriminação simples em 11 espécies. Acta Amazonica, 11(3), 653-658.

Pessotti, I., \& Otero, V.R.L. (1981). Aprendizagem em abelhas. IV: Punição e resistência à extinção. Revista Brasileira de Psicologia, 41(4), 673-680.

Reinhard, J., Srinivasan, M.V., \& Zhang, S. (2004). Scenttriggered navigation in honeybees. Nature, 427, 411. 
Rodriguez, I., Gumbert, A., de Ibarra, N.H., Hunze, J., \& Giurfa, M. (2004). Symmetry is in the eye of the "beeholder": Innate preference for bilateral symmetry in flower-naïve bumblebees. Naturwissenschaften, 91, 374377.

Srinivasan, M.V., Zhang, S.W., \& Zhu, H. (1998). Honeybees link sights to smells. Nature, 396, 637-638.

von Frisch, K. (1914). Der Farben und Formensinn der Bienen. Zoologische Jahrbücher (Physiologie), 35, 1188.

von Frisch, K. (1967). The dance language and orientation of bees. Cambridge: Belknap Press. $566 \mathrm{p}$.

Wu, W., Moreno, A.M., Tangen, J.M., \& Reinhard, J. (2013). Honeybees can discriminate between Monet and Picasso paintings. Journal of Comparative Physiology A. Neuroethology, Sensory, Neural, and Behavioral Physiology, 199(1), 45-55.

Zhang, S.W., Lehrer, M., \& Srinivasan, M.V. (1999). Honeybee memory: Navigation by associative grouping and recall of visual stimuli. Neurobiology of Learning and Memory, 72, 180-201.

Zhang, S.W., Srinivasan, M.V., \& Collett, T.S. (1995). Convergent processing in honeybee vision: Multiple channels for the recognition of shape. Proceedings of the National Academy of Sciences of the United States of America, 92, 3029-3031. 